\title{
Dipstick Urinalysis as a Test for Microhematuria and Occult Bladder Cancer
}

\author{
Richard S. Matulewicz ${ }^{\mathrm{a}, \mathrm{b}, *}$, John Oliver DeLancey ${ }^{\mathrm{a}, \mathrm{b}}$, Emily Pavey $^{\mathrm{b}}$, Edward M. Schaeffer ${ }^{\mathrm{a}}$, \\ Oana Popescu ${ }^{\mathrm{c}}$ and Joshua J. Meeks ${ }^{\mathrm{a}}$ \\ ${ }^{a}$ Department of Urology, Northwestern University Feinberg School of Medicine, Chicago, IL, USA \\ ${ }^{\mathrm{b}}$ Department of Surgery, Surgical Outcome and Quality Improvement Center (SOQIC), Northwestern \\ University Feinberg School of Medicine, Chicago, IL, USA \\ ${ }^{\mathrm{c}}$ Department of Preventative Medicine, Northwestern University Feinberg School of Medicine, \\ Chicago, IL, USA
}

\begin{abstract}
.
Introduction: There is a lack of evidence supporting the routine use of laboratory tests to detect bladder cancer. Identifying a cost-effective and widely available diagnostic aid may improve bladder cancer outcomes. We sought to evaluate the utility of dipstick urinalysis to detect microhematuria and diagnose bladder cancer in a large, diverse, contemporary cohort.

Methods: All non-pregnant women and men 35 and older with a new diagnosis of microhematuria ( $\geq 3 \mathrm{RBC} / \mathrm{hpf}$ ) were identified via a multi-center electronic medical record data warehouse query. Negative controls with no history of hematuria were randomly chosen and included to complete our cohort. Comparison between dipstick urinalysis and microscopic urinalysis on self-matched patients for the detection of microhematuria and diagnosis of bladder cancer was performed via Spearman's rank correlation coefficient, sensitivity/specificity testing, and ROC curve analysis.

Results: A total of 46,842 patients were included. Spearman's rank order correlation ( $r$ o $=0.66)$ between degree of microhematuria on dipstick urinalysis and microscopic urinalysis indicated a strong positive relationship. The ROC curve for dipstick urinalysis to identify microhematuria had an AUC of 0.80 (95\% CI $0.79-0.81)$. No difference $(p=0.83)$ in diagnostic accuracy between dipstick urinalysis (AUC $0.74,95 \%$ CI 0.70-0.78) and microscopic urinalysis (AUC 0.73, 95\% CI 0.69-0.78) as a test for bladder cancer was found.
\end{abstract}

Conclusion: Dipstick urinalysis provides a highly specific test for microhematuria and similar accuracy to microscopic urinalysis when used as a diagnostic tool to detect bladder cancer.

Keywords: urinalysis, bladder cancer, microhematuria

\section{INTRODUCTION}

Hematuria is the most common sign of bladder cancer and should prompt evaluation if blood

\footnotetext{
${ }^{*}$ Correspondence to: Richard S. Matulewicz, MD, MS, Department of Urology - Tarry 16, Northwestern University, $303 \mathrm{E}$ Chicago Ave, Chicago, IL 60611, USA. Tel.: +312 503 3238; Fax: 312908 7275; E-mail: Richard.Matulewicz@northwestern.edu.
}

is grossly visible or there are $\geq 3$ red blood cells per high-powered field (RBC/hpf) on microscopy [1]. Discovering microhematuria before it develops into gross hematuria may help identify patients at an earlier stage of bladder cancer [2]. At this time, roughly $40 \%$ of patients with bladder cancer present with advanced disease that is not amenable to curative treatment [3]. The most recent United States 
Preventative Services Task Force (USPSTF) statement on screening for bladder cancer cites a lack of evidence to recommend routine screening and noted a paucity of studies which evaluate the sensitivity, specificity, and accuracy of diagnostic tests for bladder cancer [4]. The most recent American Urologic Association (AUA) guidelines state that evaluation of microhematuria should be based solely on microscopic urinalysis and not dipstick urinalysis [5]. Dipstick urinalysis testing is widely available and less costly than more advanced laboratory tests and biomarkers. However, past bladder cancer studies assessing the utility of dipstick urinalyses have been limited by low diagnosis event rates, have focused mostly on men, and have small sample sizes [6-9].

We hypothesized that dipstick urinalysis may be as accurate as microscopic urinalysis to detect microhematuria and may represent an efficient, cost-effective way of identifying patients with bladder cancer. To further evaluate, we performed a contemporary electronic medical record based analysis of a large, diverse population sample comparing dipstick urinalysis to microscopic urinalysis.

\section{METHODS}

Outpatient, non-pregnant women and men aged 35 and older with a new diagnosis of microhematuria ( $\geq 3 \mathrm{RBC} / \mathrm{hpf}$ on microscopic urinalysis) in the absence of a benign cause were identified over four-years (8/1/2007-8/1/2011) in Northwestern's multicenter metropolitan healthcare network's enterprise data warehouse (EDW). Patients with benign and pre-existing genitourinary causes of microhematuria were identified and excluded by ICD9 coded diagnoses (i.e.- medical renal disease, urolithiasis, kidney cancer, bladder cancer, prostate cancer, BPH). Patients with positive or equivocal urine cultures for urogenital pathogens at the time of urinalysis were also excluded. Randomly selected patients with no documented history of microscopic or gross hematuria were chosen as negative controls for analysis. Demographic, dipstick, and microscopic urinalysis information was assessed and bladder cancer diagnosis was determined via ICD9 coding (188.X) in the medical record. Microscopic urinalysis was considered the gold standard diagnostic test for microhematuria. Comparison between dipstick and microscopic urinalysis was performed on self-matched patients on a single urine sample. It is practice at our institution's lab to perform dipstick and microscopic urinalysis on all specimen ordered for microscopic urinalysis.

Dipstick urinalysis for "blood" results were categorized as negative, trace, small, moderate and large. There was no standard brand of dipstick urinalysis used throughout the study. Microscopic urinalysis was categorized via RBC/hpf: 0-2 (negative), 3-10, 11-50, 51-100, and 100+. Spearman's rank order correlation was used to measure the strength of association between dipstick urinalysis and microscopic urinalysis categories for degree of microhematuria. Sensitivity, specificity, and likelihood-ratios (LR) were calculated to evaluate the performance of dipstick results compared to microscopic urinalyses. Non-parametric ROC curves were constructed and area under the curve (AUC) was determined for dipstick urinalysis as a test for microhematuria. An ROC curve comparison between dipstick and microscopic urinalysis for the detection of bladder cancer was performed via chi-squared test. This study was approved by the Northwestern University IRB (STU00201732).

\section{RESULTS}

A total of 46,842 patients met inclusion criteria and had data for analysis. Median patient age was 54 [IQR 45-65] and full demographic characteristics of the patients included are shown in Table 1. Spearman's rank order correlation coefficient for dipstick urinalysis and microscopic urinalysis $(r h o=0.66)$ indicated a strong positive relationship between the two tests when assessing degree (or severity) of microhematuria. Figure 1 demonstrates the ROC curve for dipstick urinalysis to identify microhematuria (AUC $0.80,95 \%$ CI $0.79-0.81$ ) compared against the goldstandard microscopic urinalysis where a positive test was considered 3 or more RBC/hpf. The sensitivity, specificity, and LR for dipstick and microscopic urinalyses at all threshold and category levels for the detection of bladder cancer can be found in Table 2 . The highest sensitivity for detecting bladder cancer was 0.69 for dipstick urinalysis at the "trace" threshold and 0.73 for microscopy at the $\geq 3 \mathrm{RBC} / \mathrm{hpf}$ category. Alternatively, a specificity of 0.95 and 0.99 was found for the "large" dipstick result and $>100$ $\mathrm{RBC} / \mathrm{hpf}$, respectively.

When the area under ROC curves for dipstick and microscopic urinalysis were compared, we found no difference $(p=0.83)$ in diagnostic accuracy between 
Table 1

Demographic characteristics of the study population

\begin{tabular}{|c|c|c|}
\hline & $\mathrm{n}$ & $\%$ \\
\hline \multicolumn{3}{|l|}{ Age category } \\
\hline$<50$ & 17,505 & $37.4 \%$ \\
\hline $50-59$ & 11,885 & $25.4 \%$ \\
\hline $60-69$ & 9,593 & $20.5 \%$ \\
\hline $70+$ & 7,859 & $16.8 \%$ \\
\hline \multicolumn{3}{|l|}{ Sex } \\
\hline Female & 30,043 & $64.2 \%$ \\
\hline Male & 16,764 & $35.8 \%$ \\
\hline \multicolumn{3}{|l|}{ Race } \\
\hline White (Non-Hispanic) & 24,680 & $52.7 \%$ \\
\hline White (Hispanic) & 1,103 & $2.4 \%$ \\
\hline Black & 6,600 & $14.1 \%$ \\
\hline Asian & 1,298 & $2.8 \%$ \\
\hline Other/Unknown/Not Provided & 13,161 & $28.1 \%$ \\
\hline \multicolumn{3}{|l|}{ Index Dipstick value } \\
\hline Negative & 33,750 & $72.1 \%$ \\
\hline Trace & 4,310 & $9.2 \%$ \\
\hline Small & 3,545 & $7.6 \%$ \\
\hline Moderate & 2,732 & $5.8 \%$ \\
\hline Large & 2,464 & $5.3 \%$ \\
\hline \multicolumn{3}{|l|}{ Index Microscopic } \\
\hline \multicolumn{3}{|l|}{ Urinalysis (RBC/hpf) } \\
\hline 0 to 2 & 31,681 & $67.7 \%$ \\
\hline 3 to 10 & 11,033 & $23.6 \%$ \\
\hline 11 to 50 & 2,919 & $6.2 \%$ \\
\hline 50 to 100 & 448 & $1.0 \%$ \\
\hline $100+$ & 685 & $1.5 \%$ \\
\hline \multicolumn{3}{|l|}{ Bladder Cancer Risk } \\
\hline Overall & $143 / 46,842$ & $0.3 \%$ \\
\hline With negative micro (0-2 RBC/hpf) & $39 / 31,642$ & $0.12 \%$ \\
\hline With positive micro ( $\geq 3 \mathrm{RBC} / \mathrm{hpf}$ ) & $103 / 15,085$ & $0.68 \%$ \\
\hline With negative dip & $45 / 33,750$ & $0.13 \%$ \\
\hline With positive dip (trace+) & $98 / 13,092$ & $0.75 \%$ \\
\hline
\end{tabular}

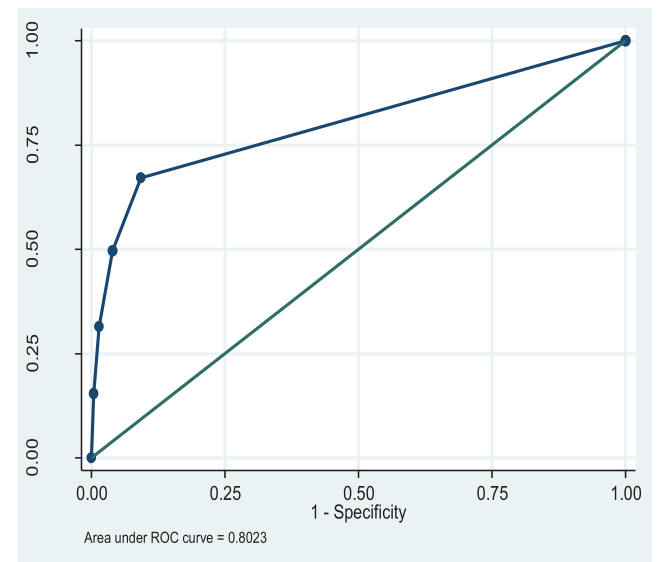

\begin{tabular}{|c|c|c|c|c|c|}
\hline Cut point & Sensitivity & Specificity & Correctly Classified & LR+ & LR- \\
\hline ( >= Negative ) & 1.000 & 0.000 & 0.32 & 1.00 & \\
\hline ( >= Trace ) & 0.671 & 0.908 & 0.83 & 7.27 & 0.36 \\
\hline ( >= Small ) & 0.497 & 0.961 & 0.81 & 12.58 & 0.52 \\
\hline ( >= Moderate ) & 0.315 & 0.986 & 0.77 & 22.45 & 0.69 \\
\hline ( >= Large ) & 0.155 & 0.996 & 0.72 & 38.92 & 0.85 \\
\hline
\end{tabular}

Fig. 1. ROC Curve for dipstick urinalysis compared to the gold standard, microscopic urinalysis. 
Table 2

Sensitivity, Specificity, Correct Classification Percentage, and Likelihood ratios for index dipstick urinalysis and index microscopic urinalysis for bladder cancer

\begin{tabular}{|c|c|c|c|c|c|}
\hline Cut point & Sensitivity & Specificity & $\begin{array}{l}\text { Classified } \\
\text { Correctly }\end{array}$ & LR+ & LR- \\
\hline & \multicolumn{4}{|c|}{ Index Dipstick UA } & \\
\hline ( $>=$ Negative $)$ & 1.00 & 0.00 & 0.00 & 1.00 & \\
\hline$(>=$ Trace $)$ & 0.69 & 0.72 & 0.72 & 2.47 & 0.44 \\
\hline$(>=$ Small $)$ & 0.58 & 0.81 & 0.81 & 3.13 & 0.52 \\
\hline ( $>=$ Moderate $)$ & 0.45 & 0.89 & 0.8 & 4.13 & 0.61 \\
\hline$(>=\mathrm{L}$ & 29 & 0.95 & 0.95 & 5.52 & 0.75 \\
\hline \multicolumn{6}{|c|}{ Index Microscopic UA } \\
\hline$(>=0$ to & 1.00 & 0.00 & 0.00 & 1.00 & \\
\hline$>=3$ to 10$)$ & 0.73 & 0.68 & 0.68 & 2.26 & 0.40 \\
\hline$=11$ to 50$)$ & 0.37 & 0.91 & 0.91 & 4.35 & 0.69 \\
\hline$>=51$ to 100$)$ & 0.1 & 0.98 & 0.97 & 7.41 & 0.84 \\
\hline$(>=100+)$ & 0.14 & 0.99 & 0.98 & 9.87 & 0.87 \\
\hline
\end{tabular}

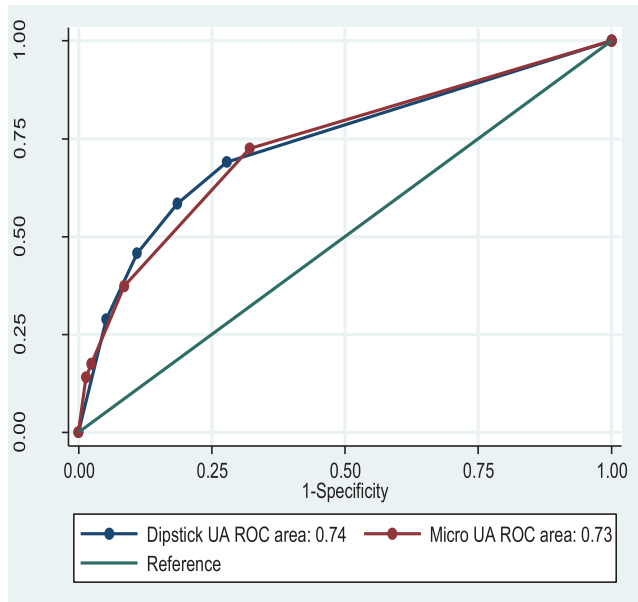

Fig. 2. Comparison of ROC curves and AUC for dipstick urinalysis and microscopic urinalysis in the diagnosis of bladder cancer.

dipstick urinalysis (AUC $0.74,95 \%$ CI $0.70-0.78$ ) and microscopic urinalysis (AUC 0.73, 95\% CI 0.69-0.78) as a test for bladder cancer (Fig. 2). Of the 33,750 patients with negative dipstick tests, 45 $(0.13 \%)$ were diagnosed with bladder cancer; 12 $(0.03 \%)$ of these patients had positive microscopic urinalyses with $\geq 3 \mathrm{RBC} / \mathrm{hpf}$.

\section{DISCUSSION}

We found a strong correlation between dipstick and microscopic urinalysis in the detection of microscopic hematuria. Furthermore, the results of dipstick and urinalysis were comparable in their ability to detect bladder cancer. Studies of microhematuriarelated urinary tract pathology based on dipstick urinalysis suffer from small sample size, are antiquated, and focus heavily on men [1]. We provide a contemporary quantitative estimate of the accuracy of dipstick urinalysis for detecting microhematuria and diagnosing bladder cancer in a large, diverse population sample. Patients are suggested to have evaluation for bladder cancer if they are found to have gross hematuria or asymptomatic microhematuria [5]. A test with high specificity will correctly identify most patients without a condition and will have few false positives. The specificity of dipstick urinalysis for bladder cancer suggests its potential for use as a tool to rule out occult pathology. Further study in high-risk populations may determine even greater utility. Though many additional clinical and risk factors should be considered when evaluating a patient at risk for bladder cancer, revisiting further prospective study of dipstick urinalysis in a large, diverse population should be considered as a means of improving bladder cancer outcomes.

We also demonstrated that the overall diagnostic accuracy of dipstick urinalysis to diagnose microhematuria when compared to microscopic urinalysis is similar and correlates strongly with degree of microhematuria. However, use of dipstick UA as a screening tool to rule in microhematuria is likely still inadequate given the low sensitivity $(0.671)$ compared to the gold standard ( $\geq 3 \mathrm{RBC} / \mathrm{hpf}$ on microscopic urinalysis) even at the most conservative thresholds. This confirms the widely held practice and belief that microscopic urinalysis should still be used as a confirmatory means of detecting microhematuria in patients with dipstick positive urine for blood and supports current guideline recommendations. Though retrospective, this would be the best supporting evidence for this practice in the literature.

This study lacks information on patient smoking status which is an important risk-factor for bladder cancer as well as clinical indications for ordering the urinalyses. Furthermore, we only assessed bladder cancer as an outcome; hematuria may be related to multiple other serious disease processes that benefit from urologic evaluation. Dipstick urinalysis results are also subject to user variability as the reagent in the stick typically changes color as a result of exposure to blood and other oxidizing agents. Additionally, the extent of microhematuria evaluation with cystoscopy and abdominopelvic imaging was not considered in diagnosis rates but likely influences rates of detection for bladder cancer. However, including only those 
patients who received a cystoscopy in the comparison between the negative and positive groups would likely confound findings since additional factors such as irritative voiding symptoms and exposures may influence decision to proceed to cystoscopy in those with and without microhematuria. In the era of unchanging bladder cancer mortality rates and rising healthcare costs, dipstick urinalysis should be re-evaluated as useful for identification of patients who warrant further work-up for microhematuria. Additional focus to determine objective factors associated with a greater risk of bladder cancer diagnosis will also help focus diagnostic evaluation strategies.

\section{CONCLUSION}

Dipstick urinalysis has a strong correlation with microscopic urinalysis when assessing degree of hematuria and similar diagnostic accuracy in determining significant microhematuria ( $\geq 3 \mathrm{RBC} / \mathrm{hpf}$ ). The high specificity of dipstick urinalysis may provide benefit when used as an inexpensive test to rule out microhematuria or bladder cancer in patients. However the low sensitivity limits its utility as a screening test. When used as a means of diagnosing bladder cancer, we found no significant difference in accuracy between dipstick urinalysis and microscopic urinalysis.

\section{REFERENCES}

[1] Nielsen M, Qaseem A. High Value Care Task Force of the American College of P. Hematuria as a Marker of Occult Urinary Tract Cancer: Advice for High-Value Care From the American College of Physicians. Ann Intern Med. 2016; 164(7):488-97. doi: 10.7326/M15-1496

[2] Ramirez D, Gupta A, Canter D, et al. Microscopic haematuria at time of diagnosis is associated with lower disease stage in patients with newly diagnosed bladder cancer. BJU International 2016;117(5):783-6.

[3] Siegel RL, Miller KD, Jemal A. Cancer statistics, 2016. CA: a Cancer Journal for Clinicians 2016;66(1):7-30.

[4] Chou R, Dana T. Screening adults for bladder cancer: A review of the evidence for the U.S. preventive services task force. Annals of Internal Medicine 2010;153(7):461-8.

[5] Davis R, Jones JS, Barocas DA, et al. Diagnosis, evaluation and follow-up of asymptomatic microhematuria (AMH) in adults: AUA guideline. The Journal of Urology 2012;188(6 Suppl):2473-81.

[6] Woolhandler S, Pels RJ, Bor DH, Himmelstein DU, Lawrence RS. Dipstick urinalysis screening of asymptomatic adults for urinary tract disorders. I. Hematuria and proteinuria. JAMA: The Journal of the American Medical Association 1989;262(9):1214-19.

[7] Messing EM, Madeb R, Young T, et al. Long-term outcome of hematuria home screening for bladder cancer in men. Cancer 2006;107(9):2173-9.

[8] Messing EM, Young TB, Hunt VB, et al. Hematuria home screening: Repeat testing results. The Journal of Urology 1995;154(1):57-61.

[9] Messing EM, Young TB, Hunt VB, et al. Comparison of bladder cancer outcome in men undergoing hematuria home screening versus those with standard clinical presentations. Urology 1995;45(3):387-96. 